\title{
Bifurcation Analysis and Solutions of a Higher-Order Nonlinear Schrödinger Equation
}

\author{
Yi Li, ${ }^{1,2}$ Wen-rui Shan, ${ }^{1,2}$ Tianping Shuai, ${ }^{1,2}$ and Ke Rao ${ }^{1,2}$ \\ ${ }^{1}$ State Key Laboratory of Information Photonics and Optical Communications, Beijing University of Posts and Telecommunications, \\ Beijing 100876, China \\ ${ }^{2}$ School of Science, Beijing University of Posts and Telecommunications, Beijing 100876, China
}

Correspondence should be addressed to Yi Li; liyi0209@sina.com

Received 2 October 2014; Revised 8 December 2014; Accepted 8 December 2014

Academic Editor: Reza Jazar

Copyright (c) $2015 \mathrm{Yi} \mathrm{Li}$ et al. This is an open access article distributed under the Creative Commons Attribution License, which permits unrestricted use, distribution, and reproduction in any medium, provided the original work is properly cited.

\begin{abstract}
The purpose of this paper is to investigate a higher-order nonlinear Schrödinger equation with non-Kerr term by using the bifurcation theory method of dynamical systems and to provide its bounded traveling wave solutions. Applying the theory, we discuss the bifurcation of phase portraits and investigate the relation between the bounded orbit of the traveling wave system and the energy level. Through the research, new traveling wave solutions are given, which include solitary wave solutions, kink wave solutions, and periodic wave solutions.
\end{abstract}

\section{Introduction}

In the past decades, communication systems have scored a great growth of the transmission capacity. Due to the undamped and unchanged characteristics in a far distance, optical solitons are the focus of many research groups during the past decades and stand a good chance to be the main information carriers in telecommunications in the future. Ignoring optical losses, the wave dynamics of nonlinear pulse propagation in a monomode fiber is described by the nonlinear Schrödinger equation $[1,2]$, which accounts for the group velocity dispersion and self-phase modulation. To increase the bit rate, it is often desirable to use shorter femtosecond pulses. However, when short pulses are considered, the equation can no longer represent the propagations of light pulses in fibers because higher-order dispersion terms and the non-Kerr nonlinearity effects cannot be neglected. This phenomenon can be expressed by a higher-order nonlinear Schrödinger equation [3]:

$$
\begin{aligned}
i E_{z} & -\frac{\beta_{2}}{2} E_{t t}+\gamma_{1}|E|^{2} E \\
& =i \frac{\beta_{3}}{6} E_{t t t}+i \alpha_{1}\left(|E|^{2} E\right)_{t}+i \alpha_{2} E\left(|E|^{2}\right)_{t},
\end{aligned}
$$

where $\alpha_{1}, \alpha_{2}, \beta_{2}, \beta_{3}$, and $\gamma_{1}$ are real constants. $E(t, x)$ is a slowly varying envelope amplitude, $t$ represents the normalized retarded time (in the group velocity frame), and $z$ represents the normalized distance along the direction of propagation. $\beta_{2}$ comes from the group velocity dispersion (GVD). $\gamma_{1}$ is proportional to the nonlinear index which originates from the Kerr effect. $\beta_{3}$ is the coefficients with the relevant work of the third-order dispersion. $\alpha_{1}$ is related to self-steepening due to stimulated Raman scattering. The coefficient of the last term that is proportional to $\alpha_{2}=\gamma_{1} T_{R}$ has its origin in the delayed Raman response $T_{R}$. Generally speaking, $T_{R}$ can be estimated from the slope of the Raman gain and is defined as the first moment of the nonlinear response function [4]. In fact, $\alpha_{2}$ should be an imaginary number, but many analytical studies have been done when $\alpha_{2}$ is real, such as Painlevé property [5], inverse scattering transform [6], Hirota direct method, and conservation laws [7]. These researches verify its integrable nature and have obtained many exact wave solutions.

Laser spectroscopic techniques have been widely used in all fields of science. It can help us observe the physical processes in materials and molecules which occur on a femtosecond time scale by using ultrashort lasers. The pulses can also be applied in telecommunication and ultrafast signal 
routing systems. Research indicates that non-Kerr nonlinear effects begin to have some effects when the pulse width becomes narrower and the intensity of the incident light field becomes stronger. The influence is described by the NLS family of equations with nonlinear terms [8]. The nonlinearity due to fifth-order susceptibility can be obtained in many optical materials such as semiconductors and some transparent organic materials. Actually, it is also important to include some additional higher-order perturbation effects into the HNLS equation to analyze the solitary wave solution in a non-Kerr nonlinear medium.

In this paper, with the aid of Mathematica, we study the new traveling wave solutions for a higher-order NLS equation that contains the non-Kerr nonlinear terms, which describes propagation of very short pulses in highly nonlinear optical fibers by using different elliptic functions. The bifurcation theory method is widely used to solve differential equations [9-12]. By using this method of dynamical systems, we obtain the explicit expressions of the bounded traveling wave solutions for the equation and investigate the relation between the bounded orbit of the traveling wave system and the energy level $h$. The new solutions correspond to the orbits on phase portraits and they include solitary, kink, and periodic wave solutions. Note that the existence of solitary wave solutions depends essentially on the model coefficients and therefore on the specific nonlinear features of the medium.

\section{Bifurcation and Phase Portraits}

We consider the higher-order NLS equation with non-Kerr term [13]:

$$
\begin{aligned}
i E_{z}-\frac{\beta_{2}}{2} E_{t t}+\gamma_{1}|E|^{2} E= & i \frac{\beta_{3}}{6} E_{t t t}+i \alpha_{1}\left(|E|^{2} E\right)_{t} \\
& +i \alpha_{2} E\left(|E|^{2}\right)_{t}-\gamma_{2}|E|^{4} E \\
& +i \alpha_{3}\left(|E|^{4} E\right)_{t}+i \alpha_{4} E\left(|E|^{4}\right)_{t},
\end{aligned}
$$

where $\alpha_{1}, \alpha_{2}, \alpha_{3}, \alpha_{4}, \beta_{2}, \beta_{3}, \gamma_{1}$, and $\gamma_{2}$ are real constants. $\gamma_{2}, \alpha_{3}$, and $\alpha_{4}$ represent the coefficients of quintic nonKerr nonlinearities. The quintic nonlinearities arise from the expansion of the refractive index of the light pulse. The polarizations induced through the susceptibilities give the cubic and quintic (non-Kerr) terms in a nonlinear Schrödinger equation. When $\gamma_{2}=\alpha_{3}=\alpha_{4}=0$, (2) reduces to (1).

Assume that (2) has the form of the exact solution

$$
E(z, t)=u(\xi) e^{i(k z-c t)}, \quad \xi=p z-q t,
$$

where $u(\xi)$ is the real-valued function and the parameters of $p, q, k$, and $c$ are real constants to be determined later.

Substituting (3) into (2) and removing the exponential term, we change (2) into the form

$$
\begin{gathered}
\frac{1}{6} \beta_{3} c^{3} u(\xi)-\frac{1}{2} i \beta_{3} c^{2} q u^{\prime}(\xi)+\frac{1}{2} \beta_{2} c^{2} u(\xi) \\
-\frac{1}{2} \beta_{3} c q^{2} u^{\prime \prime}(\xi)-i \beta_{2} c q u^{\prime}(\xi)
\end{gathered}
$$

$$
\begin{aligned}
& +\alpha_{3}(-c) u(\xi)^{5}-\alpha_{1} c u(\xi)^{3}-k u(\xi) \\
& +i p u^{\prime}(\xi)+\frac{1}{6} i \beta_{3} q^{3} u^{(3)}(\xi) \\
& -\frac{1}{2} \beta_{2} q^{2} u^{\prime \prime}(\xi)+5 i \alpha_{3} q u(\xi)^{4} u^{\prime}(\xi) \\
& +4 i \alpha_{4} q u(\xi)^{4} u^{\prime}(\xi)+3 i \alpha_{1} q u(\xi)^{2} u^{\prime}(\xi) \\
& +2 i \alpha_{2} q u(\xi)^{2} u^{\prime}(\xi)+\gamma_{2} u(\xi)^{5}+\gamma_{1} u(\xi)^{3}=0
\end{aligned}
$$

The real and imaginary parts of (4), respectively, are

$$
\begin{aligned}
& 3 q^{2}\left(\beta_{2}+\beta_{3} c\right) u^{\prime \prime}(\xi)-\left(\beta_{3} c^{3}+3 \beta_{2} c^{2}-6 k\right) u(\xi) \\
& -\left(6 \gamma_{1}-6 \alpha_{1} c\right) u(\xi)^{3}-\left(6 \gamma_{2}-6 \alpha_{3} c\right) u(\xi)^{5}=0 \\
& -\frac{1}{2} \beta_{3} c^{2} q u^{\prime}(\xi)-\beta_{2} c q u^{\prime}(\xi)+p u^{\prime}(\xi)+\frac{1}{6} \beta_{3} q^{3} u^{(3)}(\xi) \\
& +5 \alpha_{3} q u(\xi)^{4} u^{\prime}(\xi)+4 \alpha_{4} q u(\xi)^{4} u^{\prime}(\xi) \\
& +3 \alpha_{1} q u(\xi)^{2} u^{\prime}(\xi)+2 \alpha_{2} q u(\xi)^{2} u^{\prime}(\xi)=0 .
\end{aligned}
$$

Integrating (6), we can get

$$
\begin{aligned}
& \frac{1}{6} \beta_{3} q^{3} u^{\prime \prime}(\xi)+\left(-\frac{1}{2} \beta_{3} c^{2} q-\beta_{2} c q+p\right) u(\xi) \\
& \quad+\left(\alpha_{1} q+\frac{2 \alpha_{2} q}{3}\right) u(\xi)^{3}+\left(\alpha_{3} q+\frac{4 \alpha_{4} q}{5}\right) u(\xi)^{5}+n=0
\end{aligned}
$$

where $n$ is a constant. Equations (5) and (7) can be reduced to an equation if $n=0$ and

$$
\begin{gathered}
-\frac{3 q^{2}\left(\beta_{2}+\beta_{3} c\right)}{\beta_{3} q^{3} / 6}=\frac{\beta_{3} c^{3}+3 \beta_{2} c^{2}-6 k}{-(1 / 2) \beta_{3} c^{2} q-\beta_{2} c q+p} \\
=\frac{6 \gamma_{1}-6 \alpha_{1} c}{\alpha_{1} q+\left(2 \alpha_{2} q / 3\right)}=\frac{6 \gamma_{2}-6 \alpha_{3} c}{\alpha_{3} q+\left(4 \alpha_{4} q / 5\right)} .
\end{gathered}
$$

By solving (8), we get

$$
c=\frac{-15 \alpha_{3} \gamma_{1}-12 \alpha_{4} \gamma_{1}+15 \alpha_{1} \gamma_{2}+10 \alpha_{2} \gamma_{2}}{2\left(5 \alpha_{2} \alpha_{3}-6 \alpha_{1} \alpha_{4}\right)} \neq-\frac{\beta_{2}}{\beta_{3}},
$$




$$
\begin{aligned}
p=\frac{-q}{45\left(5 \alpha_{2} \alpha_{3}-6 \alpha_{1} \alpha_{4}\right)^{2}\left(\alpha_{3} \gamma_{1}-\alpha_{1} \gamma_{2}\right)} \\
\times\left(-540 \alpha_{4}^{2} \alpha_{3} \beta_{3} \gamma_{1}^{3}-375 \alpha_{2}^{2} \alpha_{3} \beta_{3} \gamma_{1} \gamma_{2}^{2}\right. \\
+900 \alpha_{2} \alpha_{4} \alpha_{3} \beta_{3} \gamma_{1}^{2} \gamma_{2}-432 \alpha_{4}^{3} \beta_{3} \gamma_{1}^{3} \\
+250 \alpha_{2}^{3} \beta_{3} \gamma_{2}^{3}+375 \alpha_{1} \alpha_{2}^{2} \beta_{3} \gamma_{2}^{3} \\
\quad-900 \alpha_{2}^{2} \alpha_{4} \beta_{3} \gamma_{1} \gamma_{2}^{2}-900 \alpha_{1} \alpha_{2} \alpha_{4} \beta_{3} \gamma_{1} \gamma_{2}^{2} \\
+540 \alpha_{1} \alpha_{4}^{2} \beta_{3} \gamma_{1}^{2} \gamma_{2}+1080 \alpha_{2} \alpha_{4}^{2} \beta_{3} \gamma_{1}^{2} \gamma_{2} \\
+750 \alpha_{2}^{3} \alpha_{3}^{3} k-2700 \alpha_{1} \alpha_{2}^{2} \alpha_{4} \alpha_{3}^{2} k \\
\left.\quad+3240 \alpha_{1}^{2} \alpha_{2} \alpha_{4}^{2} \alpha_{3} k-1296 \alpha_{1}^{3} \alpha_{4}^{3} k\right), \\
\beta_{2}=-\frac{\beta_{3}\left(-5 \alpha_{3} \gamma_{1}-6 \alpha_{4} \gamma_{1}+5 \alpha_{1} \gamma_{2}+5 \alpha_{2} \gamma_{2}\right),}{5 \alpha_{2} \alpha_{3}-6 \alpha_{1} \alpha_{4}},
\end{aligned}
$$

or

$$
k=\frac{\beta_{2}^{3}}{3 \beta_{3}^{2}}, \quad c=-\frac{\beta_{2}}{\beta_{3}}, \quad \gamma_{1}=-\frac{\alpha_{1} \beta_{2}}{\beta_{3}}, \quad \gamma_{2}=-\frac{\alpha_{3} \beta_{2}}{\beta_{3}} .
$$

Then (5) and (7) reduce to the following planar dynamic system:

$$
\begin{gathered}
\frac{d u}{d \xi}=y, \\
\frac{d y}{d \xi}=a_{1} u\left(u^{4}+a_{2} u^{2}+a_{3}\right),
\end{gathered}
$$

where $a_{1}=\left(-3 \beta_{3} c^{2} q-6 \beta_{2} c q+6 p\right) / \beta_{3} q^{3}, a_{2}=\left(6 \alpha_{1} q+\right.$ $\left.4 \alpha_{2} q\right) / \beta_{3} q^{3}$, and $a_{3}=\left(6 \alpha_{3} q+\left(24 \alpha_{4} q / 5\right)\right) / \beta_{3} q^{3}$.

Obviously, the above system (11) has the first integral

$$
H(u, y)=\frac{1}{2} y^{2}-\frac{a_{1}}{6} u^{6}-\frac{a_{1} a_{2}}{4} u^{4}-\frac{a_{1} a_{3}}{2} u^{2}=h .
$$

We suppose that

$$
M\left(u_{0}, y_{0}\right)=\left(\begin{array}{cc}
0 & 1 \\
a_{1} a_{3} & 0
\end{array}\right)
$$

is the coefficient matrix of the linearized system (11) at an equilibrium point $\left(u_{0}, y_{0}\right)$ and

$$
J\left(u_{0}, y_{0}\right)=\left[\begin{array}{cc}
0 & 1 \\
a_{1}\left(4 u_{0}^{4}+2 a_{2} u_{0}^{2}+a_{3}\right) & 0
\end{array}\right]
$$

is the Jacobian determinant. By the bifurcation theory of planar dynamical system, we know that if $J<0$, then the equilibrium point is a saddle point; if $J>0$ and $\operatorname{Trace}(M)=0$, then it is a center point; if $J>0$ and $(\operatorname{Trace}(M))^{2}-4 J=0$, then it is a node; if $J=0$ and Poincaré index of the equilibrium

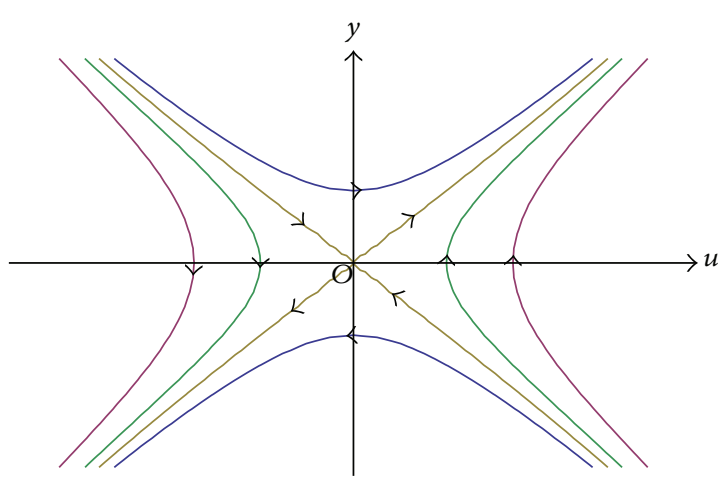

FIGURE 1: The phase portraits of (11) when $a_{1}>0$ and $a_{2}^{2}<4 a_{3}$ or $a_{1}>0, a_{2}>0, a_{3}>0$, and $a_{2}^{2}>4 a_{3}$.

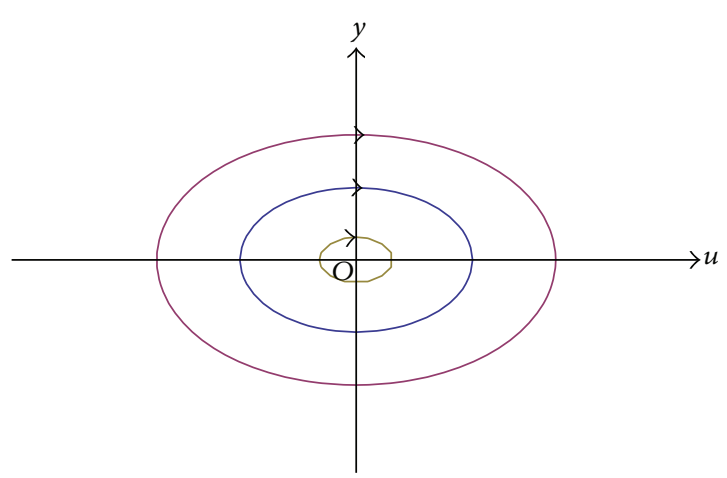

FIGURE 2: The phase portraits of (11) when $a_{1}<0, a_{2}>0, a_{3}>0$, and $a_{2}^{2}>4 a_{3}$.

point is 0 , then it is a cusp point. By using the above facts to do qualitative analysis, we have the following.

(1) If $a_{1}>0$ and $a_{2}^{2}<4 a_{3}$ or $a_{1}>0, a_{2}>0, a_{3}>0$, and $a_{2}^{2}>4 a_{3}$, then system (11) has only one equilibrium point $O(0,0)$. It is easy to find $J(O)<0$. So it is a saddle point (see Figure 1).

(2) If $a_{1}<0, a_{2}>0, a_{3}>0$, and $a_{2}^{2}>4 a_{3}$, then system (11) has only one equilibrium point $O(0,0)$. It is easy to find that $J(O)>0$ and $\operatorname{Trace}(M(O))=0$. So it is a center point (see Figure 2).

(3) If $a_{1}>0, a_{2}<0, a_{3}>0$, and $a_{2}^{2}=4 a_{3}$, then system (11) has three equilibrium points $O(0,0)$ and $P_{1 \pm}\left( \pm \sqrt{-a_{2} / 2}, 0\right) . J(O)<0, J\left(P_{1 \pm}\right)=0$, and Poincaré index of $P_{1 \pm}$ is equal to zero. So $O$ is a saddle point; $P_{1 \pm}$ are cusp points (see Figure 3 ).

(4) If $a_{1}<0, a_{2}<0, a_{3}>0$, and $a_{2}^{2}=4 a_{3}$, then system (11) has three equilibrium points $O(0,0)$ and $P_{2 \pm}\left( \pm \sqrt{-a_{2} / 2}, 0\right) . J(O)>0, \operatorname{Trace}(M(O))=0, J\left(P_{1 \pm}\right)$ $=0$, and Poincaré index of $P_{1+}$ is equal to zero. So $O$ is a center point; $P_{2 \pm}$ are cusp points (see Figure 4).

(5) If $a_{1}>0$ and $a_{3}<0$, then system (11) has three equilibrium points $O(0,0)$ and $Q_{1 \pm}\left( \pm \sqrt{\left(-a_{2}+\sqrt{a_{2}^{2}-4 a_{3}}\right) / 2}\right.$, 


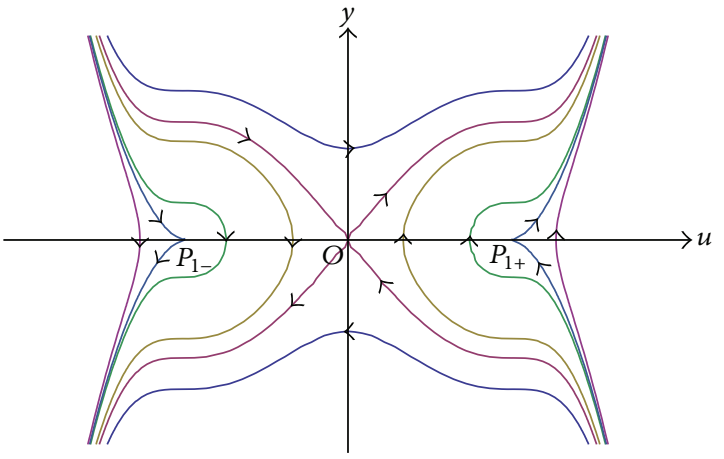

FIGURE 3: The phase portraits of (11) when $a_{1}>0, a_{2}<0, a_{3}>0$, and $a_{2}^{2}=4 a_{3}$.

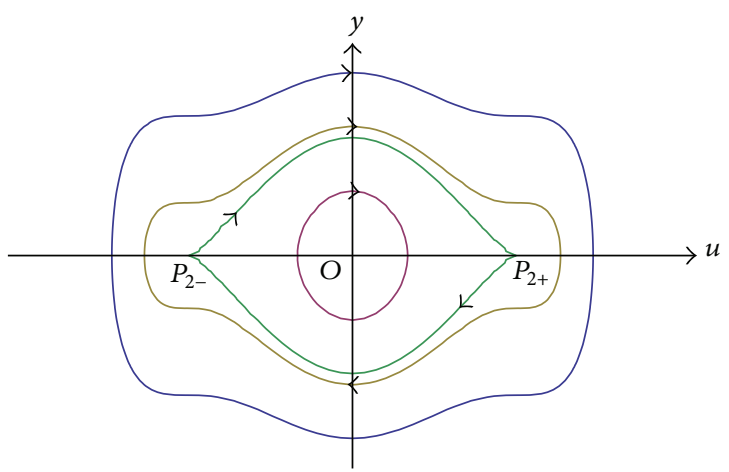

FIGURE 4: The phase portraits of (11) when $a_{1}<0, a_{2}<0, a_{3}>0$, and $a_{2}^{2}=4 a_{3}$.

0). $J(O)>0$, $\operatorname{Trace}(M(O))=0$, and $J\left(Q_{1 \pm}\right)<0$. So $O$ is a center point; $Q_{1 \pm}$ are saddle points (see Figure 5).

(6) If $a_{1}<0$ and $a_{3}<0$, then system (11) has three equilibrium points $O(0,0)$ and $Q_{2 \pm}\left( \pm \sqrt{\left(-a_{2}+\sqrt{a_{2}^{2}-4 a_{3}}\right) / 2}\right.$, $0) . J(O)<0, J\left(Q_{1 \pm}\right)>0$, and $\operatorname{Trace}\left(M\left(Q_{1 \pm}\right)\right)=0$. So $O$ is a saddle point; $Q_{2 \pm}$ are center points (see Figure $6)$.

(7) If $a_{1}>0, a_{2}<0, a_{3}>0$, and $a_{2}^{2}>4 a_{3}$, then system (11) has five equilibrium points $O(0,0), \quad R_{1 \pm}\left( \pm \sqrt{\left(-a_{2}-\sqrt{a_{2}^{2}-4 a_{3}}\right) / 2}, 0\right), \quad$ and $S_{1 \pm}\left( \pm \sqrt{\left(-a_{2}+\sqrt{a_{2}^{2}-4 a_{3}}\right) / 2}, 0\right) . J(O)<0, J\left(S_{1 \pm}\right)<0$, $J\left(R_{1 \pm}\right)>0$, and $\operatorname{Trace}\left(M\left(R_{1 \pm}\right)\right)=0$. So $O$ and $S_{1 \pm}$ are saddle points; $R_{1 \pm}$ are center points. Furthermore, if $a_{2}^{2}<(16 / 3) a_{3}$, then $H\left(R_{1 \pm}\right)<H\left(S_{1 \pm}\right)<H(O)$ (see Figure 7); if $a_{2}^{2}=(16 / 3) a_{3}$, then $H\left(R_{1 \pm}\right)<H\left(S_{1 \pm}\right)=$ $H(O)$ (see Figure 8); if $a_{2}^{2}>(16 / 3) a_{3}$, then $H\left(R_{1 \pm}\right)<$ $H(O)<H\left(S_{1 \pm}\right)$ (see Figure 9).

(8) If $a_{1}<0, a_{2}<0, a_{3}>0$, and $a_{2}^{2}>4 a_{3}$, then system (11) has five equilibrium points

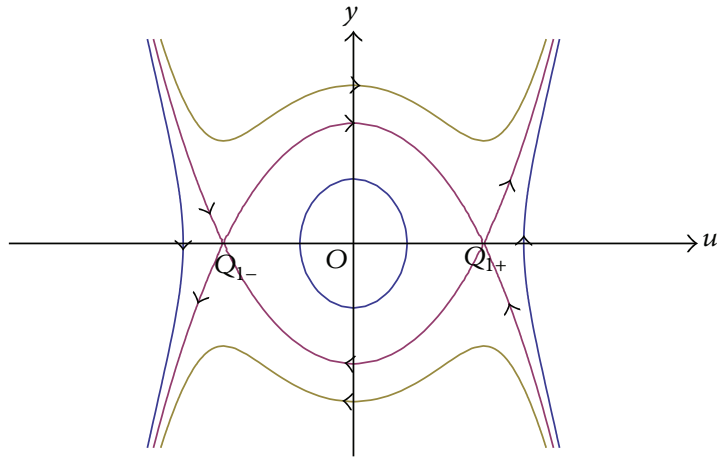

FIgURE 5: The phase portraits of (11) when $a_{1}>0$ and $a_{3}<0$.

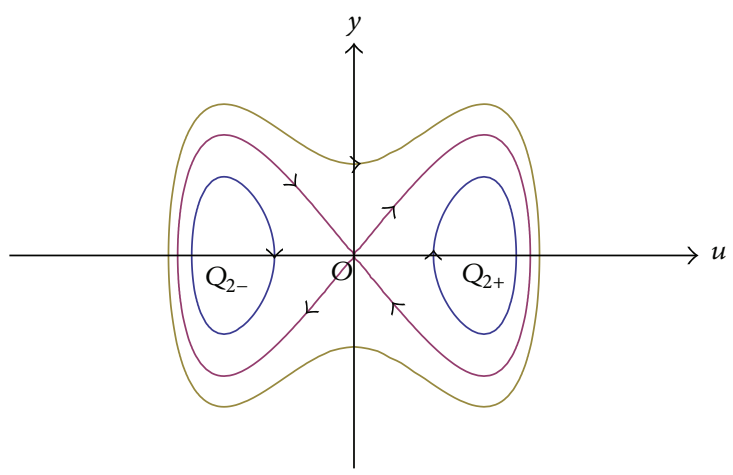

FIGURE 6: The phase portraits of (11) when $a_{1}<0$ and $a_{3}<0$.

$O(0,0), \quad R_{2 \pm}\left( \pm \sqrt{\left(-a_{2}-\sqrt{a_{2}^{2}-4 a_{3}}\right) / 2}, 0\right), \quad$ and $\left.S_{2 \pm} \pm \sqrt{\left(-a_{2}+\sqrt{a_{2}^{2}-4 a_{3}}\right) / 2}, 0\right) . \quad J(O)>0$, $\operatorname{Trace}(M(O))=0, J\left(S_{2 \pm}\right)>0, \operatorname{Trace}\left(M\left(S_{2 \pm}\right)\right)=0$, and $J\left(R_{1 \pm}\right)<0$. So $O$ and $S_{2 \pm}$ are center points; $R_{2 \pm}$ are saddle points (see Figure 10).

For a fixed $h \in R$, the curve

$$
\bar{C}_{h}=\{(u, y) \in R \times R: H(u, y)=h\}
$$

is called a level curve with energy level $h$ [14]. Obviously, each orbit of (12) is a branch of certain energy curve. For convenience, we name the orbit as the orbit with energy level $h$.

To facilitate further analysis, we investigate the relation between the bounded orbit of (12) and the energy level $h$.

Put

$$
F_{h}(u)=h+\frac{a_{1}}{6} u^{6}+\frac{a_{1} a_{2}}{4} u^{4}+\frac{a_{1} a_{3}}{2} u^{2} .
$$




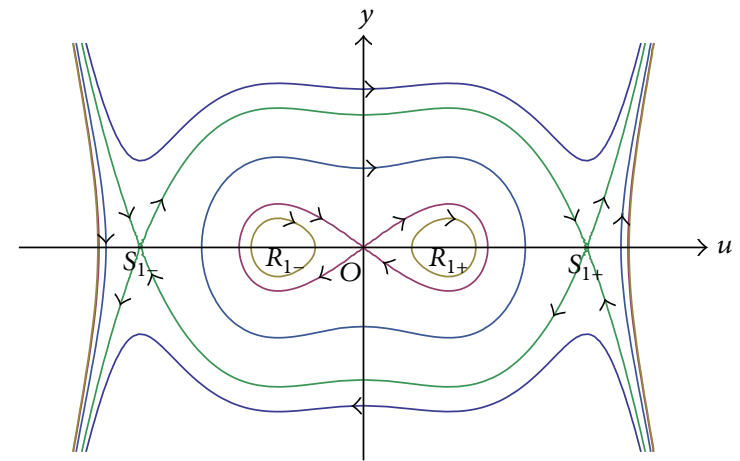

FIGURE 7: The phase portraits of (11) when $a_{1}>0, a_{2}<0, a_{3}>0$, and $a_{2}^{2}>(16 / 3) a_{3}$.

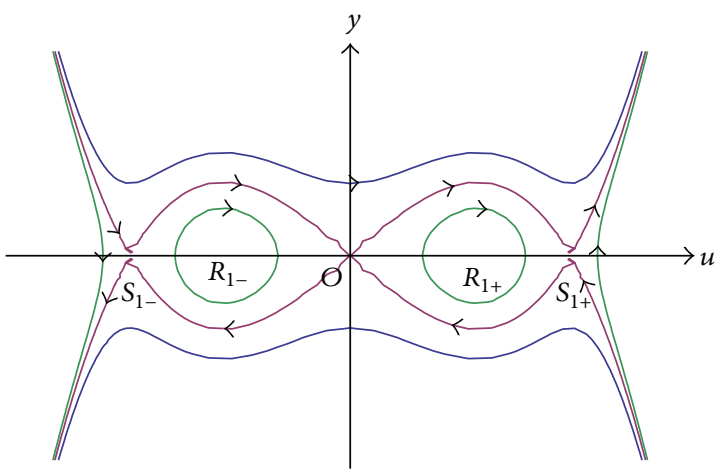

FIGURE 8: The phase portraits of (11) when $a_{1}>0, a_{2}<0, a_{3}>0$, and $a_{2}^{2}=(16 / 3) a_{3}$.

If $a_{1}>0, a_{2}<0$, and $a_{3}>0$, it is easy to obtain the five extreme points of $F_{h}(u)$ as follows:

$$
\begin{gathered}
u_{0}=0, \\
u_{1 \pm}= \pm \sqrt{\frac{-a_{2}-\sqrt{a_{2}^{2}-4 a_{3}}}{2}} \\
u_{2 \pm}= \pm \sqrt{\frac{-a_{2}+\sqrt{a_{2}^{2}-4 a_{3}}}{2}} .
\end{gathered}
$$

Let

$$
h_{1}=-F_{0}\left(u_{1 \pm}\right), \quad h_{2}=-F_{0}\left(u_{2 \pm}\right) .
$$

Therefore we can easily draw the graphics of the function $F_{h}(u)$ in Figures 11, 12, and 13.

Observe that the energy curve $\bar{C}_{h}$ is equivalent to the curve defined by $(1 / 2) y^{2}=F_{h}(u)$. According to the above analysis, we have the following.

Case 1. Assume that $a_{1}>0, a_{2}<0, a_{3}>0$, and $a_{2}^{2}>$ $(16 / 3) a_{3}$; system (11) has three saddle points $O, S_{1 \pm}$, and two center points $R_{1 \pm}$. There exist two homoclinic orbits with energy level 0 to the saddle point $O$ (corresponding to

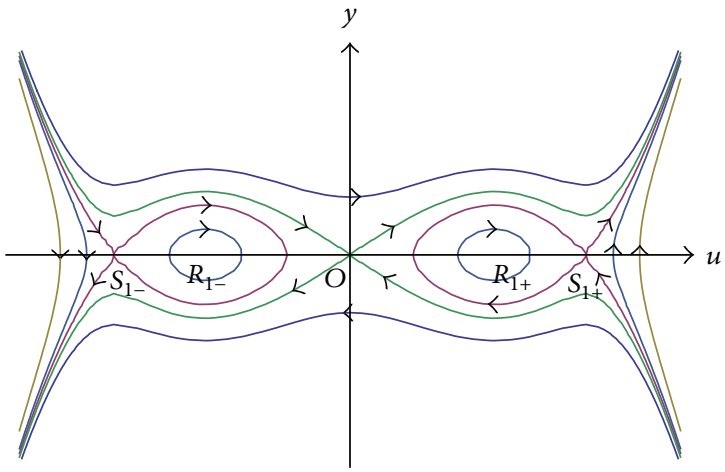

FIgURE 9: The phase portraits of (11) when $a_{1}>0, a_{2}<0, a_{3}>0$, and $4 a_{3}<a_{2}^{2}<(16 / 3) a_{3}$.

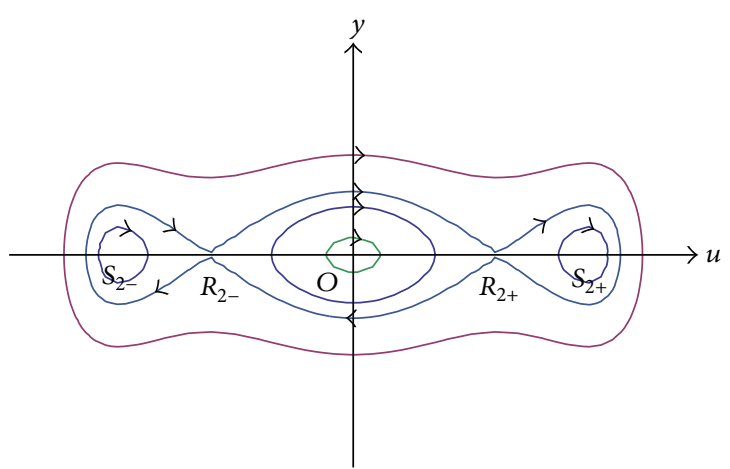

FIGURE 10: The phase portraits of (11) when $a_{1}<0, a_{2}<0, a_{3}>0$, and $a_{2}^{2}>4 a_{3}$.

red closed lines $L(O, O)$ in the left half-plane and the right half-plane in Figure 7) and two families of periodic orbits with energy level $h\left(h \in\left(h_{1}, 0\right)\right)$ around the centers $R_{1 \pm}$ (corresponding to orange closed lines in the left half-plane and the right half-plane in Figure 7) which lie inside of above two homoclinic orbits, respectively. Besides, system (11) has a singular closed orbit with energy level $h_{2}$ to the closed curve $L\left(S_{1-}, S_{1+}, S_{1-}\right)$ (corresponding to the green closed lines in Figure 7) and a family of periodic orbits with energy level $h$ $\left(h \in\left(0, h_{2}\right)\right.$ ) (corresponding to the family of periodic orbits enclosing the equilibrium points $O$ and $S_{1+}$ ) which lie inside of above singular closed orbit. It implies that for (11) there exist three families of periodic wave solutions, two solitary wave solutions, and two kink wave solutions.

Case 2. Assume that $a_{1}>0, a_{2}<0, a_{3}>0$, and $a_{2}^{2}=$ $(16 / 3) a_{3}$; system (11) has three saddle points $O, S_{1 \pm}$, and two center points $R_{1 \pm}$. There exist a singular closed orbit with energy level 0 to the closed curve $L\left(S_{1-}, 0, S_{1+}, 0, S_{1_{-}}\right)$ (corresponding to the red closed lines in Figure 8) and two families of periodic orbits with energy level $h\left(h \in\left(h_{1}, 0\right)\right)$ around the centers $R_{1 \pm}$ (corresponding to green closed lines in the left half-plane and the right half-plane in Figure 8) which lie inside of above singular closed orbit. It means that there exist two families of periodic wave solutions and four kink wave solutions. 


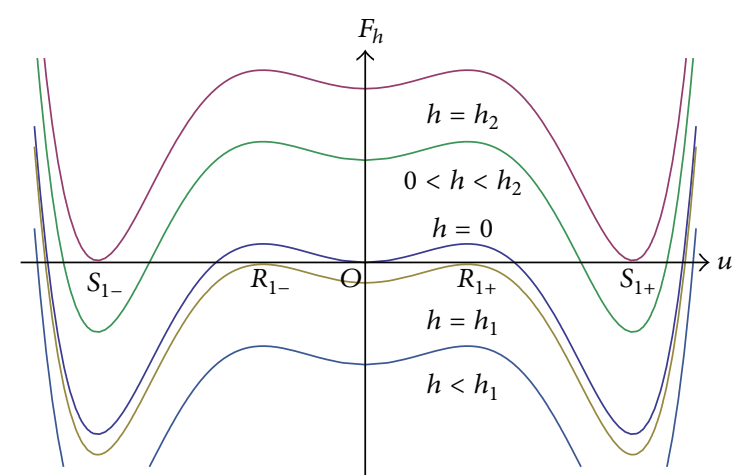

FIGURE 11: The phase portraits of (11) when $a_{1}>0, a_{2}<0, a_{3}>0$, and $a_{2}^{2}>(16 / 3) a_{3}$.

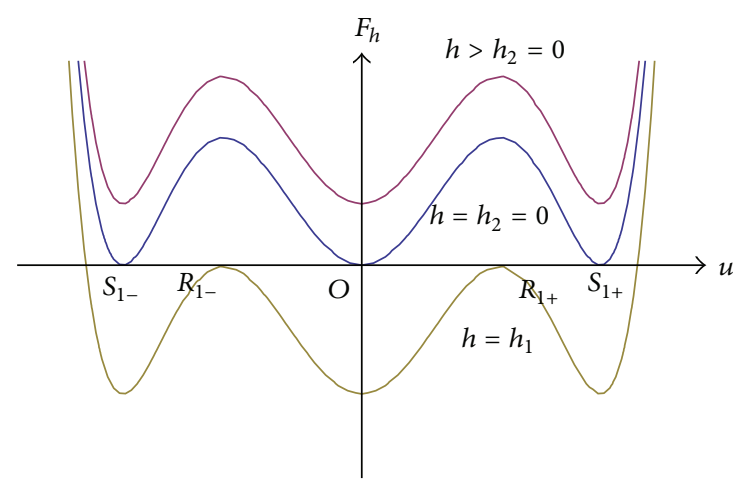

FIGURE 12: The phase portraits of (11) when $a_{1}>0, a_{2}<0, a_{3}>0$, and $a_{2}^{2}=(16 / 3) a_{3}$.

Case 3. Assume that $a_{1}>0, a_{2}<0, a_{3}>0$, and $4 a_{3}<$ $a_{2}^{2}<(16 / 3) a_{3}$; system (11) has three saddle points $O, S_{1 \pm}$, and two center points $R_{1 \pm}$. There exist two homoclinic orbits with energy level $h_{2}$ at the saddle points $S_{1 \pm}$ (corresponding to red closed lines $L\left(S_{1-}, S_{1-}\right)$ in the left half-plane and $L\left(S_{1+}, S_{1+}\right)$ in the right half-plane in Figure 9) and two families of periodic orbits with energy level $h\left(h \in\left(h_{1}, 0\right)\right)$ around the centers $R_{1 \pm}$ (corresponding to blue closed lines in the left half-plane and the right half-plane in Figure 9) which lie inside of above two homoclinic orbits, respectively.

\section{Traveling Wave Solutions of the HNLS Equation}

(1) Solitary, Kink, and Periodic Wave Solutions When $a_{1}>0$, $a_{2}<0, a_{3}>0$, and $a_{2}^{2}>(16 / 3) a_{3}$. (i) If $h=0$, as is seen in Figure 7, there are two symmetric homoclinic orbits connected at the saddle point $O$. In $(u, y)$-plane the expressions of the homoclinic orbits are given as

$$
y= \pm \sqrt{\frac{a_{1}}{3} u^{2}\left(m_{1}-u^{2}\right)\left(m_{2}-u^{2}\right)}
$$

where

$$
m_{1,2}=\frac{-3 a_{2} \mp \sqrt{9 a_{2}^{2}-48 a_{3}}}{4} .
$$

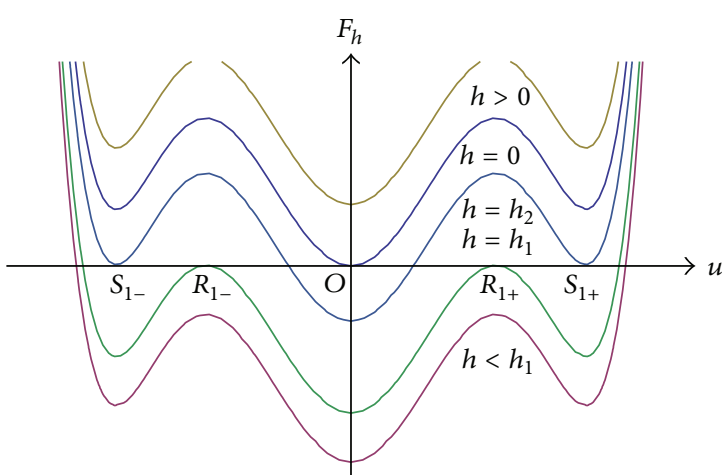

FIGURE 13: The phase portraits of (11) when $a_{1}>0, a_{2}<0, a_{3}>0$, and $4 a_{3}<a_{2}^{2}<(16 / 3) a_{3}$.

Substituting (19) into $d u / d \xi=y$ and integrating them along the homoclinic orbits, noticing that $E(z, t)=u(\xi) e^{i(k z-c t)}$ and $\xi=p z-q t$, we get two solitary wave solutions:

$$
\begin{aligned}
& E_{1 \pm}(z, t) \\
& = \pm \sqrt{\frac{m_{1} m_{2} \operatorname{sech}^{2} \sqrt{a_{1} m_{1} m_{2} / 3}(p z-q t)}{m_{2}-m_{1} \tanh ^{2} \sqrt{a_{1} m_{1} m_{2} / 3}(p z-q t)}} e^{i(k z-c t)},
\end{aligned}
$$

which correspond to the family of homoclinic orbits $L(O, O)$ in the left half-plane and $L(O, O)$ in the right half-plane shown in Figure 7.

(ii) If $h=h_{2}$, as is seen in Figure 7, there are two heteroclinic orbits connected at the saddle points $S_{1-}$ and $S_{1+}$. In $(u, y)$-plane the expressions of the heteroclinic orbits are given as

$$
y= \pm \sqrt{\frac{a_{1}}{3}\left(u^{2}-n_{1}\right)\left(n_{2}-u^{2}\right)^{2}}
$$

where

$$
\begin{aligned}
& n_{1}=\frac{-a_{2}-2 \sqrt{a_{2}^{2}-4 a_{3}}}{2}, \\
& n_{2}=\frac{-a_{2}+\sqrt{a_{2}^{2}-4 a_{3}}}{2} .
\end{aligned}
$$

Substituting (22) into $d u / d \xi=y$ and integrating them along the heteroclinic orbits, noticing that $E(z, t)=u(\xi) e^{i(k z-c t)}$ and $\xi=p z-q t$, we get two kink wave solutions:

$$
\begin{aligned}
E_{2 \pm}(z, t)= & \pm \frac{\sqrt{-n_{1} n_{2}} \tanh \sqrt{a_{1} n_{2}\left(n_{2}-n_{1}\right) / 3}(p z-q t)}{\sqrt{n_{2} \operatorname{sech}^{2} \sqrt{a_{1} n_{2}\left(n_{2}-n_{1}\right) / 3}(p z-q t)-n_{1}}} \\
& \times e^{i(k z-c t)},
\end{aligned}
$$

which correspond to the family of heteroclinic orbits $L\left(S_{1-}\right.$, $\left.S_{1+}\right)$ and $L\left(S_{1+}, S_{1-}\right)$ shown in Figure 7. 
(iii) If $h \in\left(h_{1}, 0\right)$, as is seen in Figure 7, there are two families of periodic orbits inside the homoclinic orbits. In $(u, y)$-plane the expressions of the periodic orbits are given as $\left(\varphi_{1} \in\left(\left(-a_{2}-\sqrt{a_{2}^{2}-4 a_{3}}\right) / 2, n_{1}\right)\right)$ :

$$
y= \pm \sqrt{\frac{a_{1}}{3}\left(\varphi_{1}-u^{2}\right)\left(u^{2}-\varphi_{2}\right)\left(\varphi_{3}-u^{2}\right)}
$$

where

$$
\begin{aligned}
& \varphi_{2}=\frac{-2 \varphi_{1}-3 a_{2}-\sqrt{\left(2 \varphi_{1}+3 a_{2}\right)^{2}-8\left(2 \varphi_{1}^{2}+3 a_{2} \varphi_{1}+6 a_{3}\right)}}{4}, \\
& \varphi_{3}=\frac{-2 \varphi_{1}-3 a_{2}+\sqrt{\left(2 \varphi_{1}+3 a_{2}\right)^{2}-8\left(2 \varphi_{1}^{2}+3 a_{2} \varphi_{1}+6 a_{3}\right)}}{4} .
\end{aligned}
$$

Substituting (25) into $d u / d \xi=y$ and integrating them along the periodic orbits, noticing that $E(z, t)=u(\xi) e^{i(k z-c t)}$ and $\xi=p z-q t$, we get two families of periodic wave solutions:

$$
\begin{aligned}
& E_{3 \pm}(z, t) \\
& = \pm\left(\left(\varphi_{1} \varphi_{2}\right) \times\left(\varphi_{1}-\left(\varphi_{1}-\varphi_{2}\right)\right.\right. \\
& \times \operatorname{sn}^{2}\left(\sqrt{\frac{a_{1} \varphi_{1}\left(\varphi_{3}-\varphi_{2}\right)}{3}}(p z-q t),\right. \\
& \times e^{i(k z-c t),}
\end{aligned}
$$

which correspond to two families of periodic orbits inside the homoclinic orbits in the right half-plane and in the left halfplane shown in Figure 7.

When the energy level $h \rightarrow 0$, the above periodic solutions tend to the solitary wave solutions (21).

(iv) If $h \in\left(0, h_{2}\right)$, as is seen in Figure 7, there is a family of periodic orbits enclosing the equilibrium points $R_{1-}, O$ and $R_{1+}$. In $(u, y)$-plane the expressions of the periodic orbits are given as $\left(\psi_{1} \in\left(n_{1}, m_{2}\right)\right)$ :

$$
y= \pm \sqrt{\frac{a_{1}}{3}\left(\psi_{1}-u^{2}\right)\left(u^{2}+\psi_{2}\right)\left(\psi_{3}-u^{2}\right)},
$$

where

$$
\begin{gathered}
\psi_{2}=\frac{2 \psi_{1}+3 a_{2}+\sqrt{\left(2 \psi_{1}+3 a_{2}\right)^{2}-8\left(2 \psi_{1}^{2}+3 a_{2} \psi_{1}+6 a_{3}\right)}}{4}, \\
\psi_{3}=\frac{-2 \psi_{1}-3 a_{2}+\sqrt{\left(2 \psi_{1}+3 a_{2}\right)^{2}-8\left(2 \psi_{1}^{2}+3 a_{2} \psi_{1}+6 a_{3}\right)}}{4} .
\end{gathered}
$$

Substituting (28) into $d u / d \xi=y$ and integrating them along the periodic orbits, noticing that $E(z, t)=u(\xi) e^{i(k z-c t)}$ and $\xi=p z-q t$, we get a family of periodic wave solutions:

$$
\begin{aligned}
& E_{4 \pm}(z, t) \\
& = \pm\left(\left(\psi_{1} \psi_{2}\right.\right.
\end{aligned}
$$

$$
\begin{gathered}
\times \operatorname{sn}^{2}\left(\sqrt{\frac{a_{1} \psi_{3}\left(\psi_{1}+\psi_{2}\right)}{3}}(p z-q t),\right. \\
\left.\left.\sqrt{\frac{\psi_{1}\left(\psi_{2}+\psi_{3}\right)}{\psi_{3}\left(\psi_{1}-\psi_{2}\right)}}\right)\right)
\end{gathered}
$$

$$
\times\left(\psi_{2}+\psi_{1}\right.
$$

$$
\times \mathrm{cn}^{2}\left(\sqrt{\frac{a_{1} \psi_{3}\left(\psi_{1}+\psi_{2}\right)}{3}}(p z-q t),\right.
$$

$$
\left.\left.\left.\sqrt{\frac{\psi_{1}\left(\psi_{2}+\psi_{3}\right)}{\psi_{3}\left(\psi_{1}-\psi_{2}\right)}}\right)\right)^{-1}\right)^{1 / 2}
$$

$$
\times e^{i(k z-c t)},
$$

which correspond to a family of periodic orbits enclosing three equilibrium points shown in Figure 7.

When the energy level $h \rightarrow 0$, the above periodic solutions also tend to the solitary wave solutions (21).

(2) Kink and Periodic Wave Solutions When $a_{1}>0, a_{2}<0$, $a_{3}>0$, and $a_{2}^{2}=(16 / 3) a_{3}$. (i) If $h=h_{2}$, as is seen in Figure 8, there are four heteroclinic orbits. In $(u, y)$-plane the expressions of the heteroclinic orbits are given as

$$
y= \pm \sqrt{\frac{a_{1}}{3}} u\left(u^{2}+\frac{3 a_{2}}{4}\right) .
$$

Substituting (31) into $d u / d \xi=y$ and integrating them along the heteroclinic orbits, noticing that $E(z, t)=u(\xi) e^{i(k z-c t)}$ and $\xi=p z-q t$, we get four kink wave solutions:

$$
\begin{aligned}
& E_{5 \pm}(z, t) \\
& = \pm \sqrt{\frac{\sqrt{3 a_{3}}}{2}\left[1+\tanh \sqrt{a_{1} a_{2}}\left((p z-q t)-\ln \frac{1}{\sqrt{a_{1} a_{2}}}\right)\right]} \\
& \quad \times e^{i(k z-c t)}, \\
& E_{6 \pm}(z, t) \\
& = \pm \sqrt{\frac{\sqrt{3 a_{3}}}{2}\left[1-\tanh \sqrt{a_{1} a_{2}}\left((p z-q t)+\ln \frac{1}{\sqrt{a_{1} a_{2}}}\right)\right]} \\
& \quad \times e^{i(k z-c t)},
\end{aligned}
$$




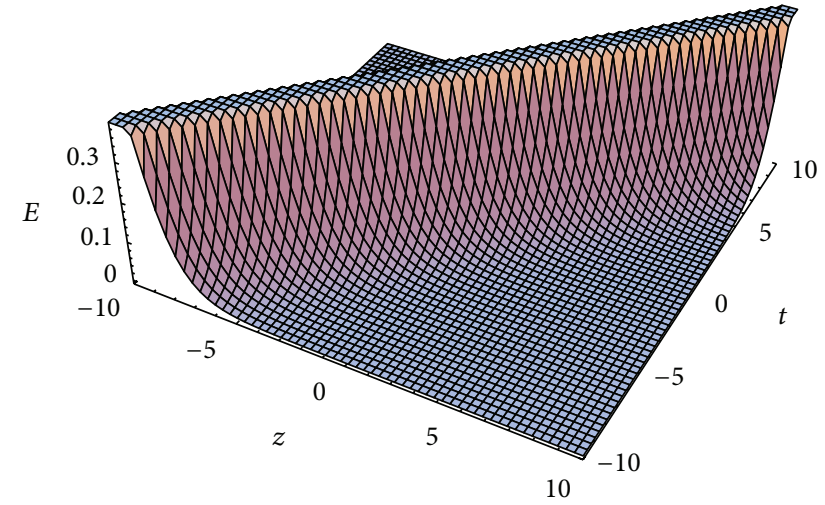

FIGURE 14: $u=\left|E_{1+}\right|$ when $a_{1}=1, a_{2}=-6, a_{3}=1, p=1$, and $q=1$.

which correspond to $L\left(O . B_{+}\right), L\left(O, B_{-}\right), L\left(B_{+}, O\right)$, and $L\left(B_{-}, O\right)$ in Figure 8.

(ii) If $h_{1}<h<h_{2}$, as is seen in Figure 8, there are two families of periodic orbits inside the homoclinic orbits. Thus we have the same solutions as (27).

(3) Solitary and Periodic Wave Solutions When $a_{1}>0, a_{2}<0$, $a_{3}>0$, and $4 a_{3}<a_{2}^{2}<(16 / 3) a_{3}$. (i) If $h=h_{2}$, as is seen in Figure 9, there are two symmetric homoclinic orbits connected at the saddle points $S_{1-}$ and $S_{1+}$. In $(u, y)$-plane the expressions of the homoclinic orbits are given as

$$
y= \pm \sqrt{\frac{a_{1}}{3}\left(u^{2}-n_{1}\right)\left(n_{2}-u^{2}\right)^{2}}
$$

where $n_{1}$ and $n_{2}$ satisfy (23).

Substituting (33) into $d u / d \xi=y$ and integrating them along the homoclinic orbits, noticing that $E(z, t)=$ $u(\xi) e^{i(k z-c t)}$ and $\xi=p z-q t$, we get two solitary wave solutions:

$$
\begin{aligned}
E_{7 \pm}(z, t) & \\
= & \pm \sqrt{\frac{n_{1} n_{2}}{n_{2}+\left(n_{1}-n_{2}\right) \tanh ^{2} \sqrt{a_{1} n_{2}\left(n_{2}-n_{1}\right) / 3}(p z-q t)}} \\
& \times e^{i(k z-c t)} .
\end{aligned}
$$

(ii) If $h \in\left(h_{1}, h_{2}\right)$, as is seen in Figure 9, there are two families of periodic orbits inside the homoclinic orbits. Thus we have the same solutions as (27).

We will draw the figures of some solutions under the special conditions. Figure 14 is a bell solitary wave solution of the field $\left|E_{1+}\right|$ given by (21) when the energy level $h=0$. Figure 15 shows the kink wave solution of the field $\left|E_{2+}\right|$ given by (21) when the energy level $h=h_{2}$. Figure 16 is a plot for the periodic wave solution of the field $\left|E_{3+}\right|$ given by (27) when the energy level $h=-0.0390$. Figure 17 is a periodic wave solution of the field $\left|E_{4+}\right|$ given by (30) when the energy level $h=0.0030$. Figure 18 shows periodic wave solutions of the

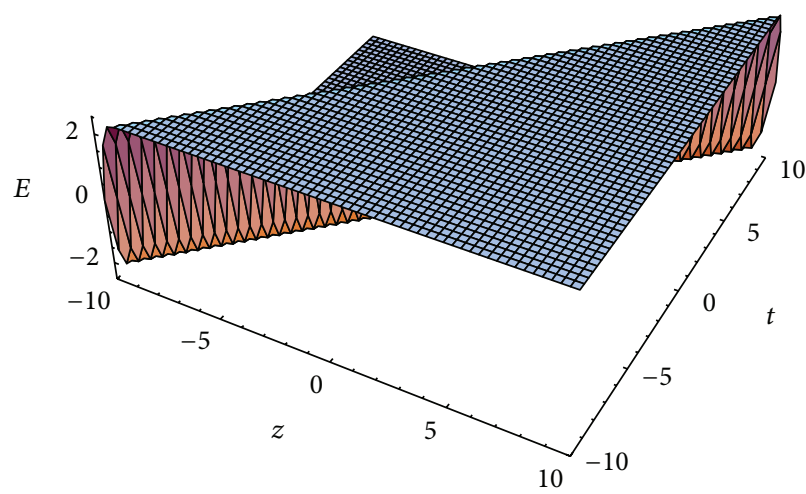

Figure 15: $u=\left|E_{2+}\right|$ when $a_{1}=1, a_{2}=-6, a_{3}=1, p=1$, and $q=1$.

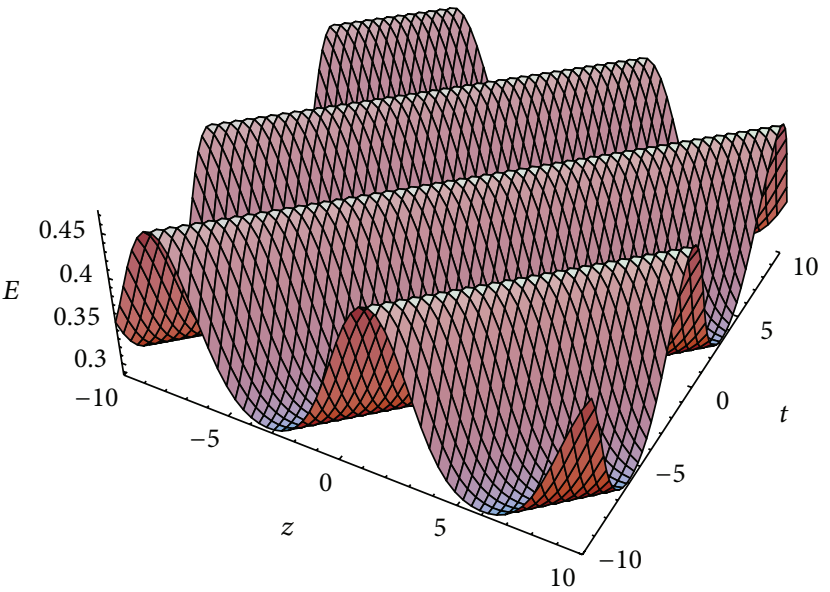

Figure 16: $u=\left|E_{3+}\right|$ when $a_{1}=1, a_{2}=-6, a_{3}=1, p=1, q=1$, and $h=-0.0390$.

field $\left|E_{3+}\right|$ given by (27) when $\varphi_{1} \in\left(\left(-a_{2}-\sqrt{a_{2}^{2}-4 a_{3}}\right) / 2, n_{1}\right)$. We can see that when $\varphi_{1} \rightarrow\left(-a_{2}-\sqrt{a_{2}^{2}-4 a_{3}}\right) / 2$, or we say $h \rightarrow h_{1}$, these periodic wave solutions will be reduced back to zero. When $\varphi_{1} \rightarrow n_{1}$, or we say $h \rightarrow 0$, the periodic solutions tend to the solitary wave solutions (21). Figure 19 is a periodic wave solution of the field $\left|E_{4+}\right|$ when $\phi_{1}$ changes. When the energy level $h \rightarrow 0$, the above periodic solutions also tend to the solitary wave solutions (21). Figures 20, 21, $22,23,24$, and 25 show the relationship near critical points between $E_{1+}(\xi), E_{2+}(\xi)$ and $a_{1}, a_{2}$, and $a_{3}$, respectively.

\section{Conclusion}

By using the bifurcation theory method of dynamical systems, we successfully obtain 10 phase portraits for the corresponding dynamic system of (2). Through analysing three of these phase portraits, we get new traveling wave solutions, including solitary wave solutions, kink wave solutions, and periodic wave solutions. The solutions are new and have not been investigated. By the dependent variable transformations, four linear forms for (2) can also be obtained. We will 


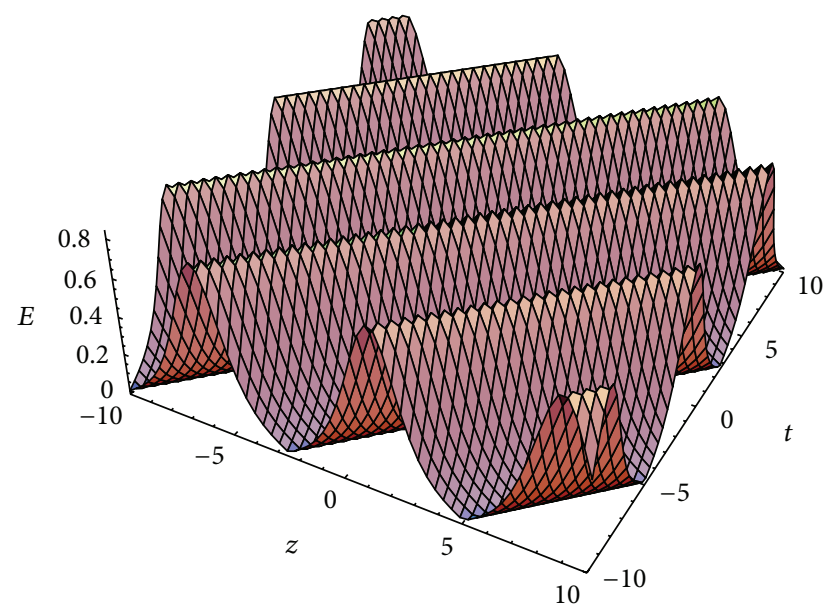

FIGURE 17: $u=\left|E_{4+}\right|$ when $a_{1}=1, a_{2}=-6, a_{3}=1, p=1, q=1$, and $h=0.0030$.

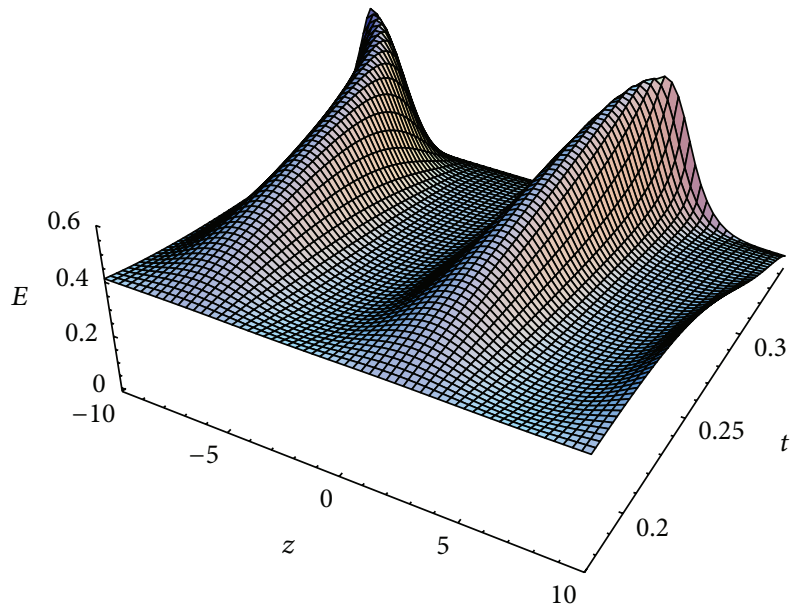

Figure 18: $u=\left|E_{3+}\right|$ when $a_{1}=1, a_{2}=-6$, and $a_{3}=1$.

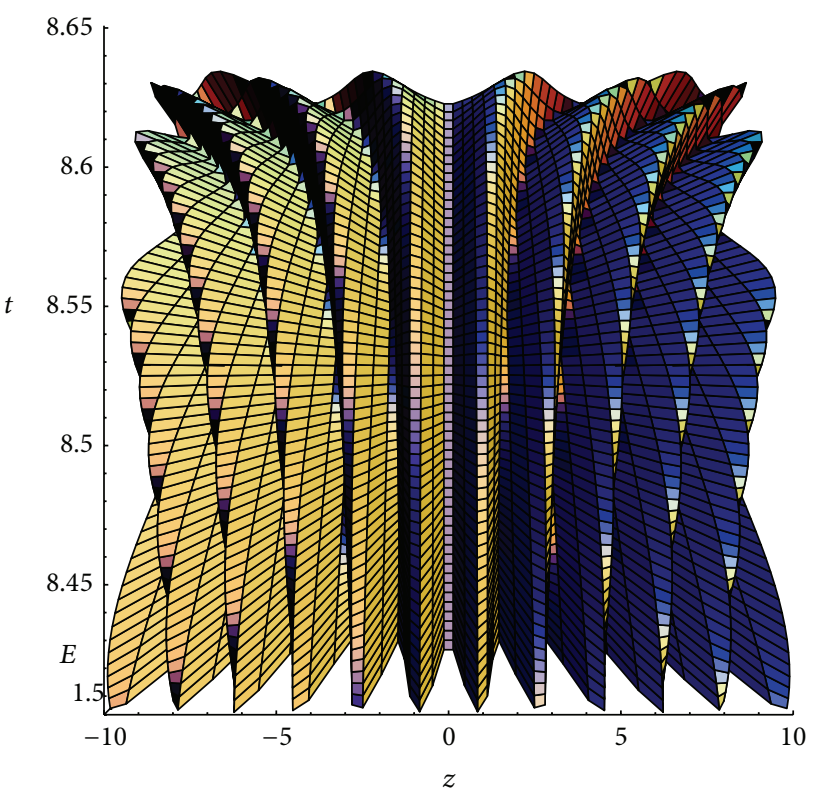

FIGURE 19: $u=\left|E_{4+}\right|$ when $a_{1}=1, a_{2}=-6$, and $a_{3}=1$.

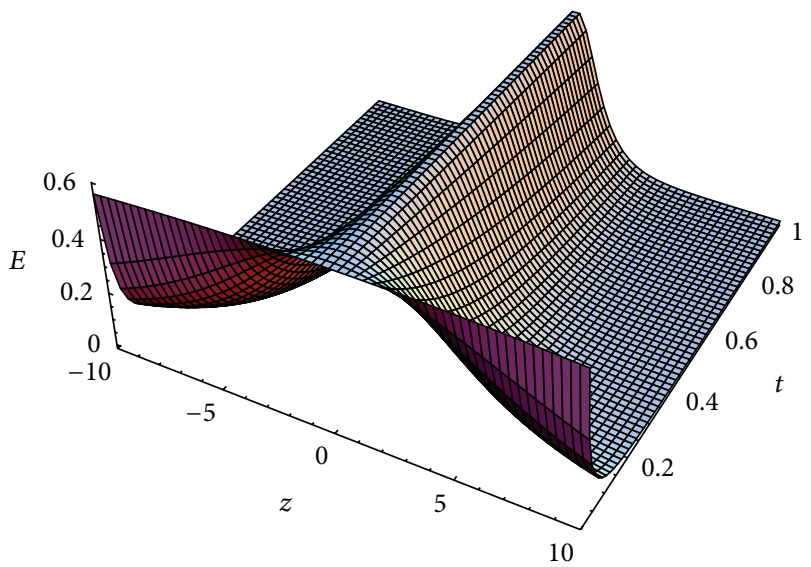

Figure 20: $u=\left|E_{1+}\right|$ when $a_{2}=-6$ and $a_{3}=1$.

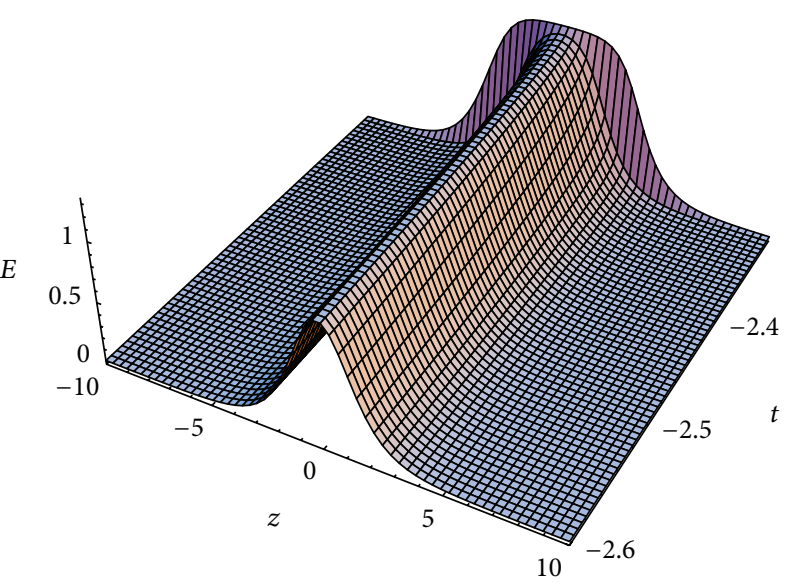

FIGURE 21: $u=\left|E_{1+}\right|$ when $a_{1}=1$ and $a_{3}=1$.

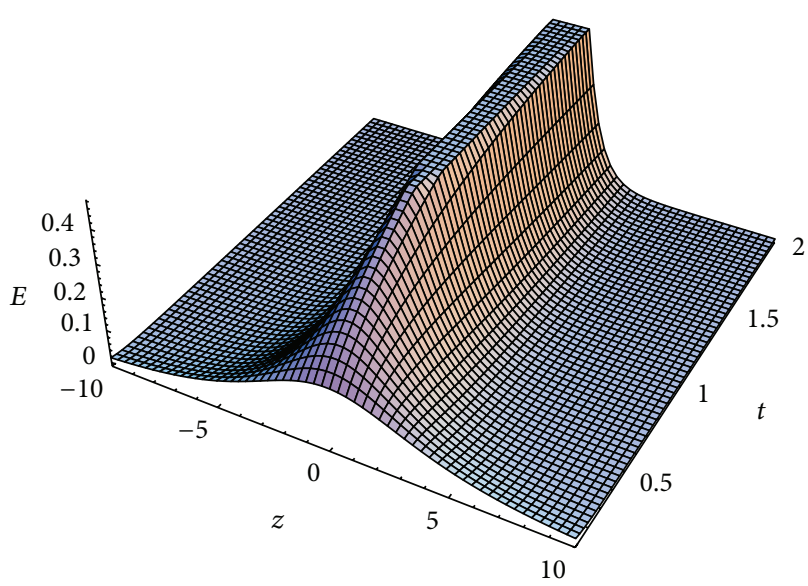

FIGURE 22: $u=\left|E_{2+}\right|$ when $a_{1}=1$ and $a_{2}=-6$.

continue to consider the problem by using Hirota method in the future. 


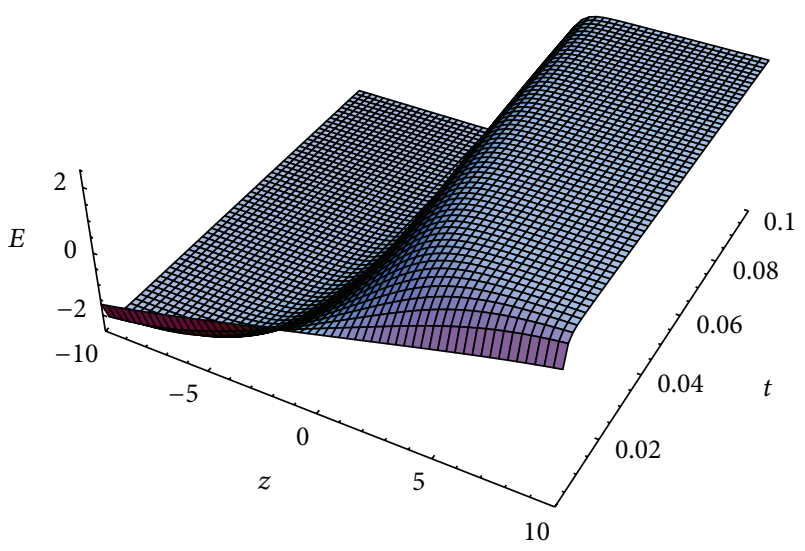

Figure 23: $u=\left|E_{2+}\right|$ when $a_{2}=-6$ and $a_{3}=1$.

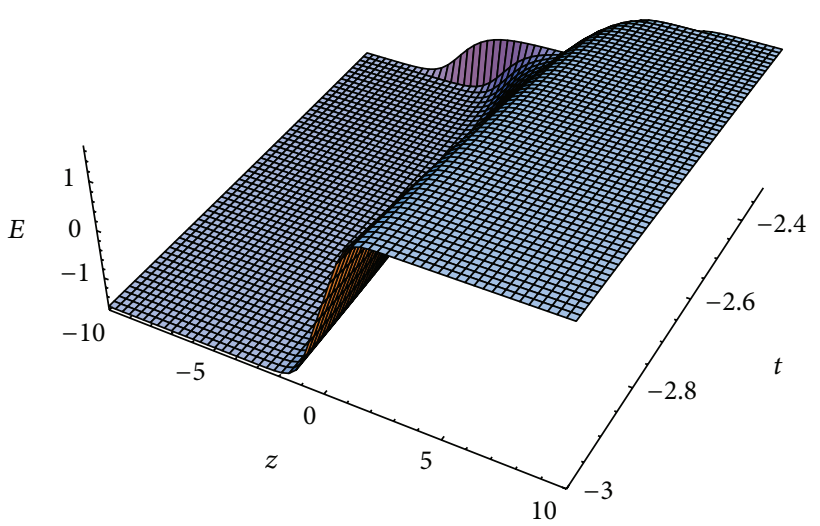

FIGURE 24: $u=\left|E_{2+}\right|$ when $a_{1}=1$ and $a_{3}=1$.

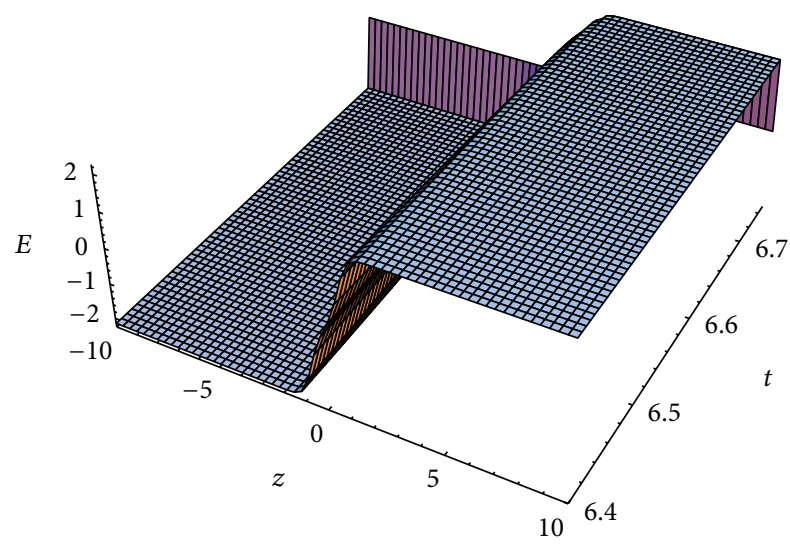

FIGURE 25: $u=\left|E_{1+}\right|$ when $a_{1}=1$ and $a_{2}=-6$.

\section{Conflict of Interests}

The authors declare that there is no conflict of interests regarding the publication of this paper.

\section{Acknowledgments}

The authors express their sincere thanks to the editor and reviewers for their valuable comments. This work has been supported by the National Natural Science Foundation of China under Grant no. 11272023, by the Open Fund of State Key Laboratory of Information Photonics and Optical Communications (Beijing University of Posts and Telecommunications) under Grant no. IPOC2013B008, and by the Fundamental Research Funds for the Central Universities of China under Grant no. 2011BUPTYB02.

\section{References}

[1] G. P. Agrawal, Nonlinear Fiber Optics, Academic Press, San Diego, Calif, USA, 1995.

[2] A. Hasegawa and Y. Kodama, Solitons in Optical Communications, Oxford University Press, Oxford, UK, 1995.

[3] Y. Kodama and A. Hasegawa, "Nonlinear pulse propagation in a monomode dielectric guide," IEEE Journal of Quantum Electronics, vol. 23, no. 5, pp. 510-524, 1987.

[4] G. P. Agrawal, Nonlinear Fiber Optics, Academic Press, New York, NY, USA, 2006.

[5] K. Porsezian and K. Nakkeeran, "Optical solitons in presence of Kerr dispersion and self-frequency shift," Physical Review Letters, vol. 76, no. 21, pp. 3955-3958, 1996.

[6] S. Palacios, A. Guinea, J. Fernández-Díaz, and R. D. Crespo, "Dark solitary waves in the nonlinear Schrödinger equation with third order dispersion, self-steepening, and self-frequency shift," Physical Review E, vol. 60, no. 1, pp. R45-R47, 1999.

[7] W.-J. Liu, "Soliton interaction in the higher-order nonlinear Schrödinger equation investigated with Hirota's bilinear method," Physical Review E, vol. 77, Article ID 066605, 2008.

[8] R. Radhakrishnan, A. Kundu, and M. Lakshmanan, "Coupled nonlinear Schrödinger equations with cubic-quintic nonlinearity: integrability and soliton interaction in non-Kerr media," Physical Review E-Statistical Physics, Plasmas, Fluids, and Related Interdisciplinary Topics, vol. 60, no. 3, pp. 3314-3323, 1999.

[9] Z. Zhang, F.-L. Xia, and X.-P. Li, "Bifurcation analysis and the travelling wave solutions of the Klein-Gordon-Zakharov equations," Pramana, vol. 80, no. 1, pp. 41-59, 2013.

[10] M. Song, B. S. Ahmed, and A. Biswas, "Topological soliton solution and bifurcation analysis of the Klein-Gordon-Zakharov equation in $(1+1)$-dimensions with power law nonlinearity," Journal of Applied Mathematics, vol. 2013, Article ID 972416, 7 pages, 2013.

[11] M. Song, Z. Liu, E. Zerrad, and A. Biswas, "Singular solitons and bifurcation analysis of quadratic nonlinear Klein-Gordon equation," Applied Mathematics \& Information Sciences, vol. 7, no. 4, pp. 1333-1340, 2013.

[12] Z. S. Wen, "Bifurcation of solitons, peakons, and periodic cusp waves for theta-equation," Nonlinear Dynamics, vol. 77, no. 1-2, pp. 247-253, 2014.

[13] A. Choudhuri and K. Porsezian, "Higher-order nonlinear Schrödinger equation with derivative non-Kerr nonlinear terms: a model for sub-10-fs-pulse propagation," Physical Review A, vol. 88, Article ID 033808, 2013.

[14] J. B. Li and H. H. Dai, On the Study of Singular Nonlinear Traveling Wave Equations: Dynamica System Approach, Science Press, Beijing, China, 2007. 


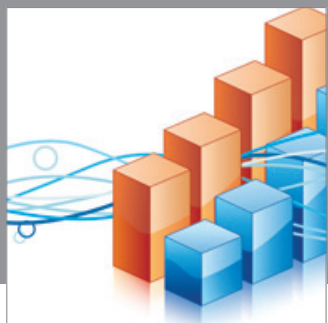

Advances in

Operations Research

mansans

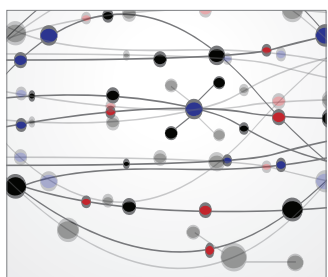

The Scientific World Journal
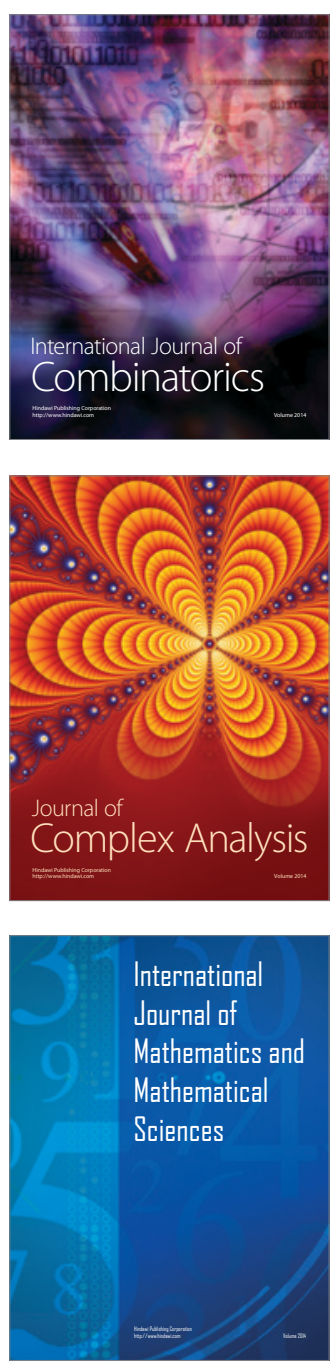
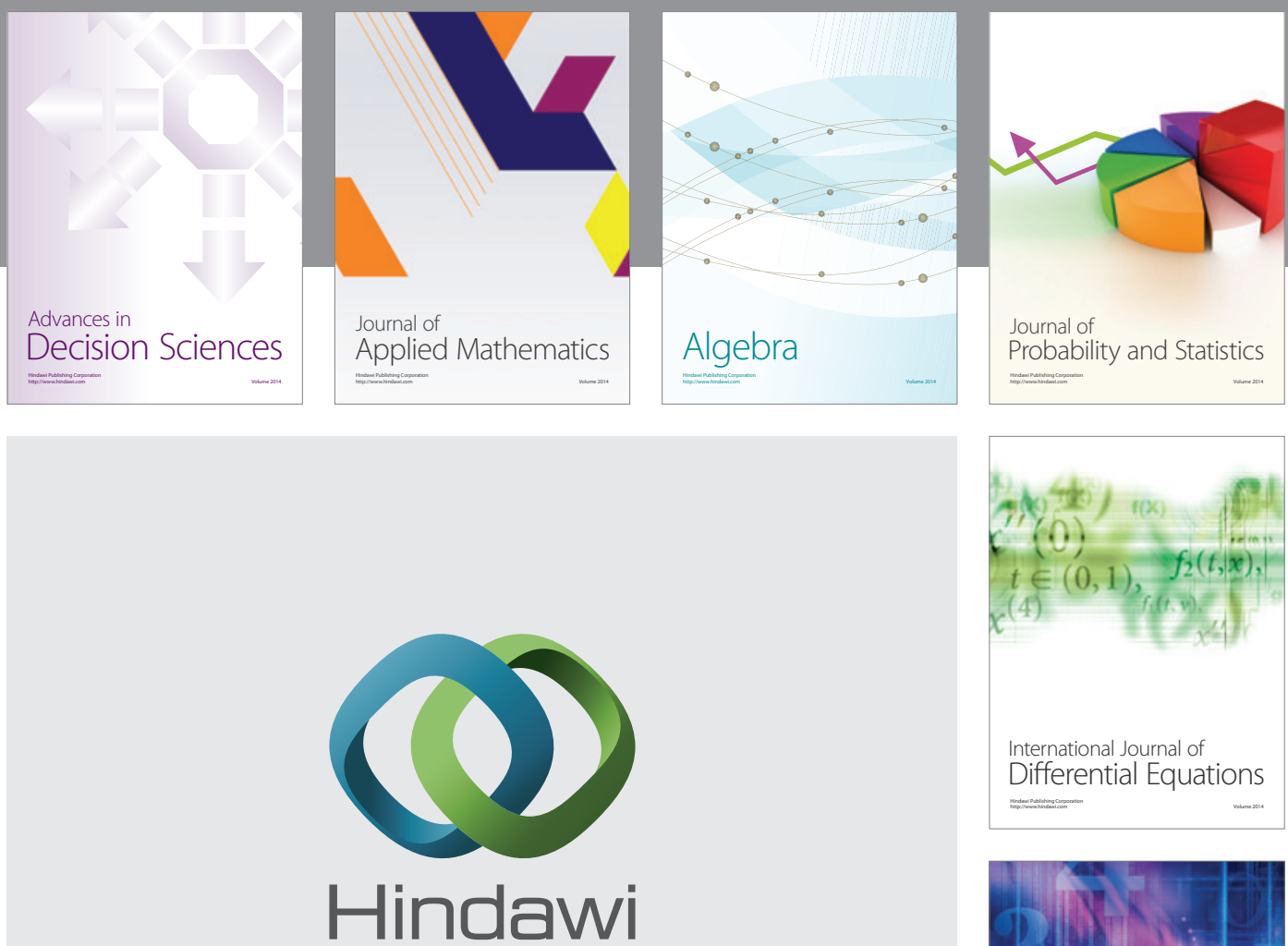

Submit your manuscripts at http://www.hindawi.com
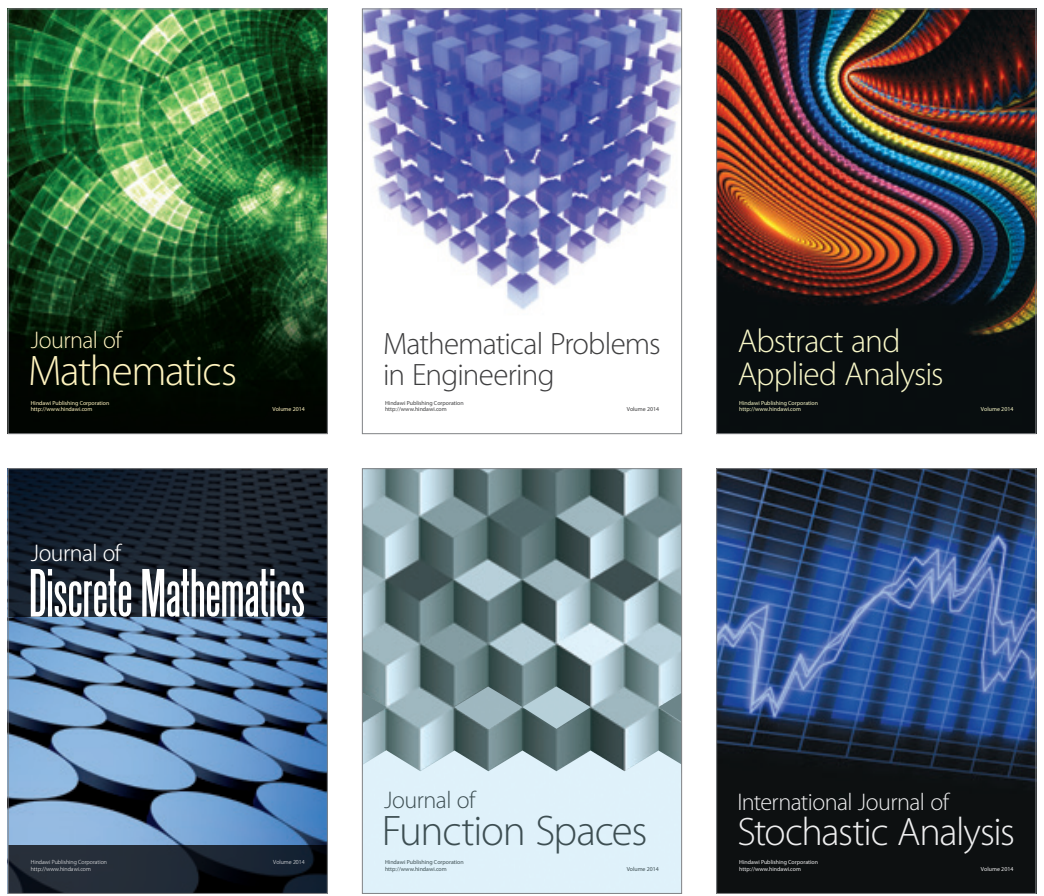

Journal of

Function Spaces

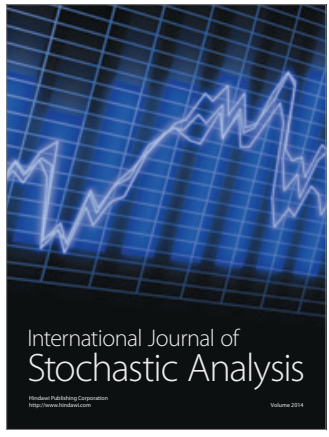

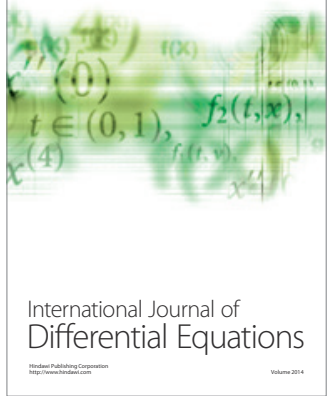
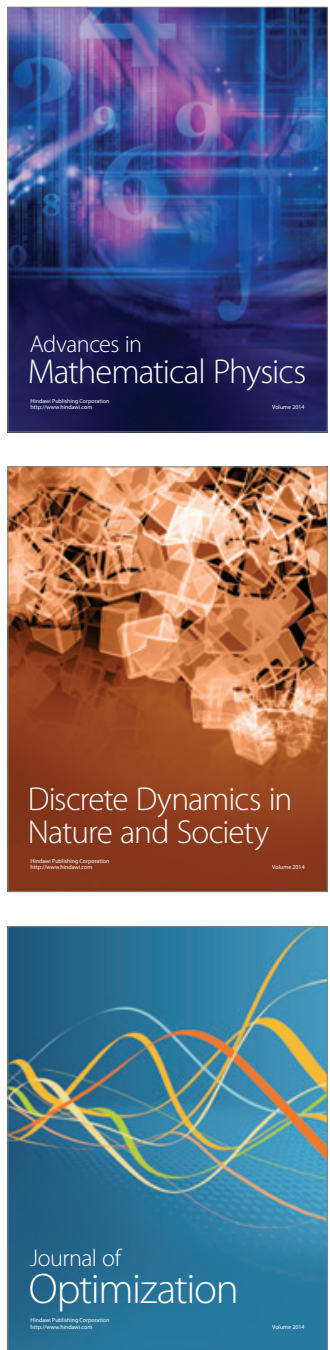